\title{
Residues and enantioselective behavior of cyflumetofen from apple production
}

\author{
Rui Quan ${ }^{\mathrm{a}, 1}$, Minmin Li ${ }^{\mathrm{a}, \mathrm{d}, 1}$, Yongguo Liuc, Nuo Jin ${ }^{\mathrm{a}}$, Jia Zhang ${ }^{\mathrm{a}}$, Ruixing $\mathrm{Li}^{\mathrm{a}}$, Fengzhong Wang ${ }^{\mathrm{a}}$, \\ Zhidong Wang ${ }^{\mathrm{a}}$, Frédéric Francis ${ }^{\mathrm{d}}$, Zhiqiang Kong ${ }^{\mathrm{a}, \mathrm{b}, *}$, Bei Fan ${ }^{\mathrm{a}, *}$ \\ ${ }^{a}$ Key Laboratory of Agro-products Quality and Safety Control in Storage and Transport Process, Ministry of Agriculture and Rural Affairs/Institute of Food Science and \\ Technology, Chinese Academy of Agricultural Sciences, Beijing 100193, PR China \\ ${ }^{\mathrm{b}}$ State Key Laboratory for Biology of Plant Diseases and Insect Pests, Institute of Plant Protection, Chinese Academy of Agricultural Sciences, Beijing 100193, PR China \\ ${ }^{\mathrm{c}}$ Beijing Key Laboratory of Flavor Chemistry, Beijing Technology and Business University (BTBU), Beijing 100048, PR China \\ ${ }^{\mathrm{d}}$ Functional and Evolutionary Entomology, Gembloux Agro-Bio-Tech, University of Liège, Passage des Déportés 2, 5030 Gembloux, Belgium
}

\section{A R T I C L E I N F O}

\section{Keywords:}

Chiral cyflumetofen

Apple processing

$\mathrm{UPC}^{2}$-MS/MS

Processing factors

Enantioselective behavior

\begin{abstract}
A B S T R A C T
The effect of food processing on the level and fate of chiral pesticide residues in apple products has rarely been investigated. In this study, we used ultra-performance convergence chromatography coupled with triple quadrupole mass spectrometry to determine the content of the novel chiral acaricide cyflumetofen. The matrixmatched calibration lines were constructed for apple slices, juice, wine and vinegar, and the determination coefficients $\left(\mathrm{r}^{2}\right)$ exceeded 0.9954 . Acceptable average recoveries were within $81.1 \%$ to $119.9 \%$, and the relative standard deviations (RSDs) ranged from $0.8 \%$ to $11.0 \%$. Processing effectiveness is represented by the processing factor (PF). The results indicated that the PFs of different procedures (washing, peeling, enzymolysis, fermentation, among others.) were generally less than 1. The reduction of cyflumetofen enantiomers during fermentation was in accordance with first-order kinetics, and stereoselective behavior was observed. This study provides reliable references for the risk assessment of cyflumetofen in the processing of apple products.
\end{abstract}

\section{Introduction}

Apples are a widely consumed commodity in China and the rest of the world. In 2016, the annual planting area of apples in China was approximately 2.47 million ha, with a total output of more than 40 million tons (Liu \& Zhao, 2018). Moreover, apples are one of the most popular fruits among American consumers and the apple industry is worth $\$ 3.1$ billion in the US market. However, apples are also considered to be one of the foods with the highest pesticide residues because they are vulnerable to pests and diseases throughout their cultivation cycle and require multiple phytoprotection applications per year. The Environmental Working Group (EWG), a non-profit advocacy agency, once again listed apples in its 2019 "Dirty Dozen" list. "An apple a day usually keeps the doctor away-unless it's ridden with pesticides" (Lunder, 2016), and increased risk to the health of consumers is a primary and immediate problem of toxicology. As a result, considerable attention has been paid to monitoring trace levels of pesticide residues in apples and their processing products.
The chiral pesticide cyflumetofen is a novel acaricide of benzoyl acetonitrile that is applied to apples for red spider mite control. Currently, studies on cyflumetofen focus on the mechanism of its activity and toxicity (Sun, Pang, Zhou, \& Jiao, 2016; Yoshida et al., 2012), the establishment of enantiomeric separation methods (Chen et al., 2013; Li et al., 2012; Liu, Dong, Xu, et al., 2016), and risk assessment (Guo et al., 2018; Wang et al., 2016). However, relatively few studies on stereoselective degradation in the processing of agricultural products have been reported. Yoshida et al. (2012) fed rats with fodder containing cyflumetofen for 90 days in chronic toxicity tests, and found that the kidneys and livers of rats were enlarged, and adrenocortical cells were vacuolized. The selective degradation behavior of cyflumetofen in the course of citrus planting was studied by Sun et al. (2016); the results showed that (-)-cyflumetofen was degraded preferentially in citrus with a half-life of 16.48 days and the enantiomer fraction (EF) value was 0.42 . In general, there are substantial differences among the enantiomers of chiral pesticides in terms of toxicity, biological activity, metabolism, and degradation behavior because interactions between

* Corresponding authors at: Key Laboratory of Agro-products Quality and Safety Control in Storage and Transport Process, Ministry of Agriculture and Rural Affairs/Institute of Food Science and Technology, Chinese Academy of Agricultural Sciences, Beijing 100193, PR China (Z. Kong).

E-mail addresses: kongzhiqiang@caas.cn (Z. Kong), fanbeicaas@163.com (B. Fan).

${ }^{1}$ These authors contributed equally to this paper. 
chiral compounds and enzymes are different (Liu et al., 2015).

Apples are consumed raw, and also in the form of apple sauce, juice, concentrates, wine, vinegar, or slices, depending on their seasonality, low durability, and low disease resistance. In particular, apple vinegar and wine not only reduce cholesterol levels and improve antioxidant capacity, but also help regulate metabolism (Seydim, Guzel-Seydim, Doguc, Savas, \& Budak, 2016). Complex physical, chemical, and biological changes are involved during different kinds of apple processing. In addition, there is little doubt that pesticide residues in agricultural products directly affect the quality of processed products. Food processing can change the concentration, distribution, and chemical properties of pesticides present on fruits and vegetables, increase and reduce the quantity of pesticide residues in the final product, and even promote the production of highly toxic pesticide metabolites (Keikotlhaile, Spanoghe, \& Steurbaut, 2010). Currently, extensive research has suggested that processing has a considerable impact on the behavior and fate of pesticides. Rasmusssen, Poulsen, and Hansen (2003) found that storage resulted in significant degradation of kresoxim-methyl, fenitrothion, diazinon, tolylfluanid and chlorpyrifos (from 25 to 69\%) during home processing of apples, and boiling reduced fenitrothion and tolylfluanid residue levels by $32 \%$ and $81 \%$, respectively. Han et al. (2013) found that peeling and coring reduced spirotetramat-enol and spirotetramat concentrations by $62.9 \%$ and $76.4 \%$ from apples, respectively. Similarly, juicing, sterilizing, and enzymolysis also contribute to reductions in pesticide residue levels ( $\mathrm{Li}$ et al., 2015). To date, few studies have focused on effects of the fermentation process. Kong et al. (2016) showed that dichlorvos and malathion residues degraded partially with prolonged fermentation during beer processing. The reason for the reduction of pesticides during fermentation may be related to effects of pesticides on microbial activities, and in turn, the latter degrade or absorb pesticides partly.

PFs are generally used to demonstrate the impact and disposition of food processing on pesticide residues. The European Food Safety Authority (EFSA) stipulated that processing factors (PFs) are calculated and evaluated as follows: PFs = (Residue in processed fraction)/ (Residue in raw agricultural commodity) (Scholz et al., 2018). PF values $<1$ (= reduction factor) indicate decreased residual pesticide levels during processing, whereas values $>1$ ( $=$ concentration factor) indicate increased residual pesticides after processing. A PF value of 1 suggests that processing has no significant effect on pesticide degradation. PFs are of great importance in assessing the risks associated with pesticide residue intake. The PF value depends on several parameters associated with three main aspects: First, the chemical structure, physico-chemical properties, and mechanism of action of pesticides. Water solubility, molecular mass, polarity, and boiling point properties of pesticides can affect their biological activity. Pesticides with high polarity, high solubility in water, low octanol-water coefficient, contact action, and accumulation on the plant layer are more easily eliminated when they undergo processing. However, the removal of pesticides that penetrate into plant tissues by absorption, or active or passive transport is difficult. Second, the species and biological properties of processed fruits or vegetables influence PF values. Fruits and vegetables vary in morphology, mass, size, chemical composition, among other qualities, which may play an essential role in the distribution and position of pesticides in the plant. Phospholipid bilayers contain hydrophilic groups that show affinity for polar pesticides but hydrophobicity towards non-polar pesticides (Jankowska, Łozowicka, \& Kaczyński, 2019). Third, the technological conditions of food processes also influence $\mathrm{PF}$ values. Some mechanical processes affect pesticide residues. For example, squeezing can increase pesticide residues in juices when compared with of raw materials, because the top layer of a vegetable or fruit is comprised of a lipophilic membrane, which accumulates nonpolar pesticides (Rasmusssen et al., 2003). Ultrasonic waves can produce mechanical energy in the form of impulsive waves in water, which can lead to the degradation of pesticides in pores on the surface of fruits. Thermal processing can cause volatilization, hydrolysis, or degradation of pesticides, resulting in lower concentrations (Holland, Hamilton, Ohlin, \& Skidmore, 1994). Apple processing involves complex physical and chemical changes. Meanwhile, the existence of a wax layer and other factors also affect the distribution of pesticides in apple products. Consequently, it is crucial to understand the levels and behaviors of pesticide residues during apple processing, primarily whether the selective behavior of chiral compounds occurs in the process of processing and fermentation. Thus PFs will provide more precise references for risk evaluation and toxicological assessment.

To date, research on the enantiomers of cyflumetofen has been based on ultra-performance liquid chromatography coupled with tandem mass spectrometry (UPLC-MS/MS) (Liu, Dong, Chen, et al., 2016), gas chromatography-tandem mass spectrometry (GC-MS/MS) (Li et al., 2012) and ultra-performance convergence chromatography coupled with triple quadrupole mass spectrometry (UPC ${ }^{2}$-MS/MS) (Guo et al., 2018). Chen et al. (2013) detected cyflumetofen residues in several fruits and vegetables using high-performance liquid chromatography (HPLC). GC-MS/MS was also used to detect cyflumetofen residues in samples such as soil and water; however, these methods have the limitations of long retention times, low efficiency, relatively poor separation, and large organic solvent requirements (Li et al., 2012). The $\mathrm{UPC}^{2}$-MS/MS method utilizes supercritical fluid carbon dioxide as the primary mobile phase, which is more environmentally-friendly than are organic solvent-based methods (Geng et al., 2014). UPC ${ }^{2}$-MS/MS combines the advantages of supercritical fluid chromatography (SFC) and MS technologies, which could significantly reduce the analysis times and solvent consumption, increase sensitivity and separation efficiency, and improve peak shape. The enantiomeric separation and detection of chiral cyflumetofen were performed together using $\mathrm{UPC}^{2}$ MS/MS in this experiment.

Therefore, in this research, we investigated the stereoselective dissipation of cyflumetofen enantiomers in the processing of apple slices, apple juice, apple wine, and vinegar, and determined the PFs for each method, including washing, peeling, color-protecting, blanching, puffing drying, enzymolysis, and fermentation. The results obtained will provide a basis for evaluating apple products safety regarding cyflumetofen enantiomer residues.

\section{Materials and methods}

\subsection{Chemicals and reagents}

The analytical standard cyflumetofen (97\%) was obtained from Otsuka Agri Techno Co., Ltd. (Osaka, Japan). Commercial-grade 20\% cyflumetofen suspension concentrate (SC) was purchased from Jiangsu FMC Corporation (Jiangsu, China). High-purity $\mathrm{CO}_{2}(\geq 99.999 \%)$ and $\mathrm{N}_{2}$ ( $\left.\geq 99.999 \%\right)$ were obtained from Beijing Yongsheng Gas Technology Co., Ltd. (Beijing, China). Ultra-pure water was prepared using a Milli-Q reagent water system (Millipore, Burlington, MA, USA). HPLC-grade methanol, isopropanol, and acetonitrile were acquired from Fisher Scientific (Shanghai, China). Analytical-grade sodium chloride $(\mathrm{NaCl})$ and anhydrous magnesium sulfate $\left(\mathrm{MgSO}_{4}\right)$ were purchased from Sinopharm Chemical Reagent Co., Ltd. Primary secondary amine (PSA, $40 \mu \mathrm{m}$ ), graphitized carbon black (GCB, $40 \mu \mathrm{m}$ ), and $\mathrm{C}_{18}$ were acquired from DIKMA (Beijing, China).

Standard stock solutions $(1000 \mathrm{mg} / \mathrm{L})$ of racemic cyflumetofen were prepared in pure acetonitrile. The standard working solutions and calibration of racemic cyflumetofen at 50, 100, 500, 1000, 3000, and $4000 \mu \mathrm{g} / \mathrm{L}$ were prepared by successive dilution of the stock solution in pure acetonitrile. Correspondingly, (+)- and (-)-cyflumetofen were prepared at the same concentration on the day of the analysis. The matrix-matched standard solutions $(10-5000 \mu \mathrm{g} / \mathrm{L})$ were prepared by adding apple slice, apple juice, apple wine, and apple vinegar extracts to each serially diluted standard solution. All standard solutions were protected from light by storing in brown reagent bottles and wrapping the bottles with aluminum foil; the bottles were placed in a refrigerator 
at $4{ }^{\circ} \mathrm{C}$ before the analysis, and prepared samples are stable for up to three months.

\subsection{Field trials}

Field trials were performed at an orchard located in Changping District, Beijing, from July to October 2017. The orchards were examined and confirmed to be free of previous cyflumetofen exposure. Through the Organization for Economic Co-operation and Development (OECD), commercial $20 \%$ cyflumetofen SC was sprayed under sunny and windless conditions at five-fold concentrations of the recommended dosage to ensure sufficient initial precipitates of pesticides for subsequent processing and degradation studies (OECD, 2008). Cyflumetofen SC was evenly applied on leaves (both sides), fruit surfaces, trunks, and branches of the targeted apple trees until it started to form droplets on the fruit surface and drip. The pesticide was applied four times using the above method at 15-day intervals, and approximately $80 \mathrm{~kg}$ of apple samples were harvested 7 days after the last spraying. Then, these samples were transported to the laboratory in plastic bags and stored in a $4{ }^{\circ} \mathrm{C}$ refrigerator for processing.

\subsection{Sample preparation}

The apple processing experiments included four processed products: apple slices, apple juice, apple wine, and apple vinegar (Fig. 1). Approximately $10 \mathrm{~kg}$ of apples was used in each process. Classically, the same processing steps including washing, peeling, coring, slicing, and color-protecting were followed. The apple slices were first processed as group A. The sliced apple samples were soaked in $2 \%$ ascorbic acid solution for $30 \mathrm{~min}$ and immersed below the liquid level during the period to ensure full color-protection, and they were then blanched in boiling water at $100{ }^{\circ} \mathrm{C}$ for $60 \mathrm{~s}$, drained, and set aside. Next, the moisture content was dried to approximately $30 \%$ in a blast drying oven, and then the samples were placed in self-sealing bags and stored at $4{ }^{\circ} \mathrm{C}$ for $24 \mathrm{~h}$ so that the water content of the apple slices was homogenous. Finally, the samples were dried in a puffing dryer (Guangzhou Xu Zhong Food Machinery Co., Ltd., China) for $2 \mathrm{~h}$, and the final apple slices were obtained.

In this study, the same procedures were carried out for apple juice, apple wine, and apple vinegar sample preparation, as follows: colorprotecting, juicing, enzymatic treatment, filtering and clarification, sugar and acidity regulation, and sterilization. First, ascorbic acid or citric acid was added as a color fixative to the apple samples, and then the samples were squeezed using an automatic juice extractor (Zhejiang Supor Co., Ltd., China). The apple pulp was treated with pectinase ( $50 \mathrm{~g} / \mathrm{L}$ ) for enzyme treatment for $0.5 \mathrm{~h}$. Subsequently, $2 \mathrm{~g} / \mathrm{L}$ bentonite was added to the enzyme-treated juice for clarification. Twenty-four hours later, the supernatant was separated by filtration and subjected to sterilization at $135{ }^{\circ} \mathrm{C}$ for approximately $10 \mathrm{~s}$ through ultra-heat treatment (UHT) (Shanghai Kelao Machinery and Equipment Co., Ltd., China). Finally, apple juice was obtained as group B. Four grams of active dry yeast and acetic acid bacteria were respectively added to $20 \%$ sucrose solution with even stirring, and then the mixture was activated in an electro thermostatic water bath at $37{ }^{\circ} \mathrm{C}$ for $20-30 \mathrm{~min}$ until a large number of bubbles were produced. The sterilized apple juice was then transferred to a closed fermentation tank, which was inoculated with Saccharomyces cerevisiae and fermented at $25 \pm 2{ }^{\circ} \mathrm{C}$ for 7 days to obtain apple wine as group $\mathrm{C}$, as well as inoculated with Acetobacter aceti and fermented at $35 \pm 2{ }^{\circ} \mathrm{C}$ for 7 days to produce apple vinegar as group $\mathrm{D}$.

To analyze the effect of different processing steps on the selective degradation of cyflumetofen enantiomers, samples (fresh apples, washed apples, peeled apples, color-protected apples, boiled apples, blanched apples, dried apples, hydrolyzed pulp, fermented wine, and vinegar) were regularly collected after each step to determine the level and variation of cyflumetofen in the course of processing.

\subsection{Analytical methods}

Sample extraction and instrumental conditions were employed following validated and accepted residue analysis methods. The extraction of pesticides from the sample was based on the QuEChERS (Quick, Easy, Cheap, Effective, Rugged and Safe) principle (Anastassiades, Lehotay, \& Štajnbaher, 2002). Chiral separation and analysis of cyflumetofen enantiomers were performed with an Acquity UPC $^{2}$ system (Waters, Milford, MA, USA), which included a binary solvent manager, convergence manager, sample manager-FL, a Waters 515 compensation pump, and a column manager equipped with a Trefoil AMY1 $(150 \mathrm{~mm} \times 2.1 \mathrm{~mm}, 2.5 \mu \mathrm{m}$ particle size; Waters, USA). Enantiomeric detection and quantitation of cyflumetofen were achieved on a triple quadrupole Xevo-TQD mass spectrometer equipped with an ESI source (Waters, USA), wherein a positive mode was applied. Details of the sample extraction and purification scheme and instrument conditions are presented in Fig. 2. All parameter conditions are used for subsequent research studies. Masslynx V.4.1 was utilized to analyze the collected data.

\subsection{Method validation}

Pesticide residue analysis method is validated to prove that the separation method can be performed by other laboratories according to predetermined steps, and the results obtained by this method can achieve the required accuracy and precision. The method was verified, and its performance was evaluated with reference to the conventional verification program, which includes assessing calibration curve linearity, limit of detection (LOD), limit of quantification (LOQ), matrix effects, accuracy, and precision (Porter, Ward, \& Bell, 1988).

\subsection{Data analysis}

One-way ANOVA analysis was carried out with SPSS 17.0 software to calculate the half-lives of the two enantiomers. All experiments were repeated in triplicate, and the calculated values were expressed in the form of the mean \pm standard deviation. Based on the following equations, the half-lives of enantiomers in different matrices were measured (Pan et al., 2018).

$C=C_{0} \mathrm{e}^{-k t}$

where $C_{0}$ is the initial concentration $(\mathrm{mg} / \mathrm{kg}$ ) of the enantiomer, $C$ indicated the concentration $(\mathrm{mg} / \mathrm{kg})$ at time $t$ (day), and $k$ is the pesticide degradation rate constant $\left(\right.$ day $^{-1}$ ).

The selective dissipation of cyflumetofen enantiomers during processing was studied by estimating the $\mathrm{EF}$ following the equation: $\mathrm{EF}=(+) /[(+)+(-)]$, where $(+)$ and $(-)$ indicate the concentration of the specific cyflumetofen enantiomer, respectively. EF varies from 0 to 1 , while the $\mathrm{EF}$ of the racemic mixture is 0.5 . The results are presented as charts using Origin 8.0 software (Origin Lab, Northampton, MA, USA) for analysis.

\section{Results and discussion}

\subsection{Analytical method validation}

A six-point standard solution in acetonitrile was prepared at concentrations of 50,100, 500, 1000, 3000, and $4000 \mu \mathrm{g} / \mathrm{L}$, and the calibration curves were obtained by drawing peak areas in accordance with the corresponding concentrations to assess the linearity of the method. In this study, the linearity of the calibration curve of the blank matrix (apple slices, apple juice, apple wine and apple vinegar) extract was analyzed at concentrations of $10-5000 \mu \mathrm{g} / \mathrm{L}$. Satisfactory linearity was obtained for cyflumetofen enantiomers with the coefficients of 


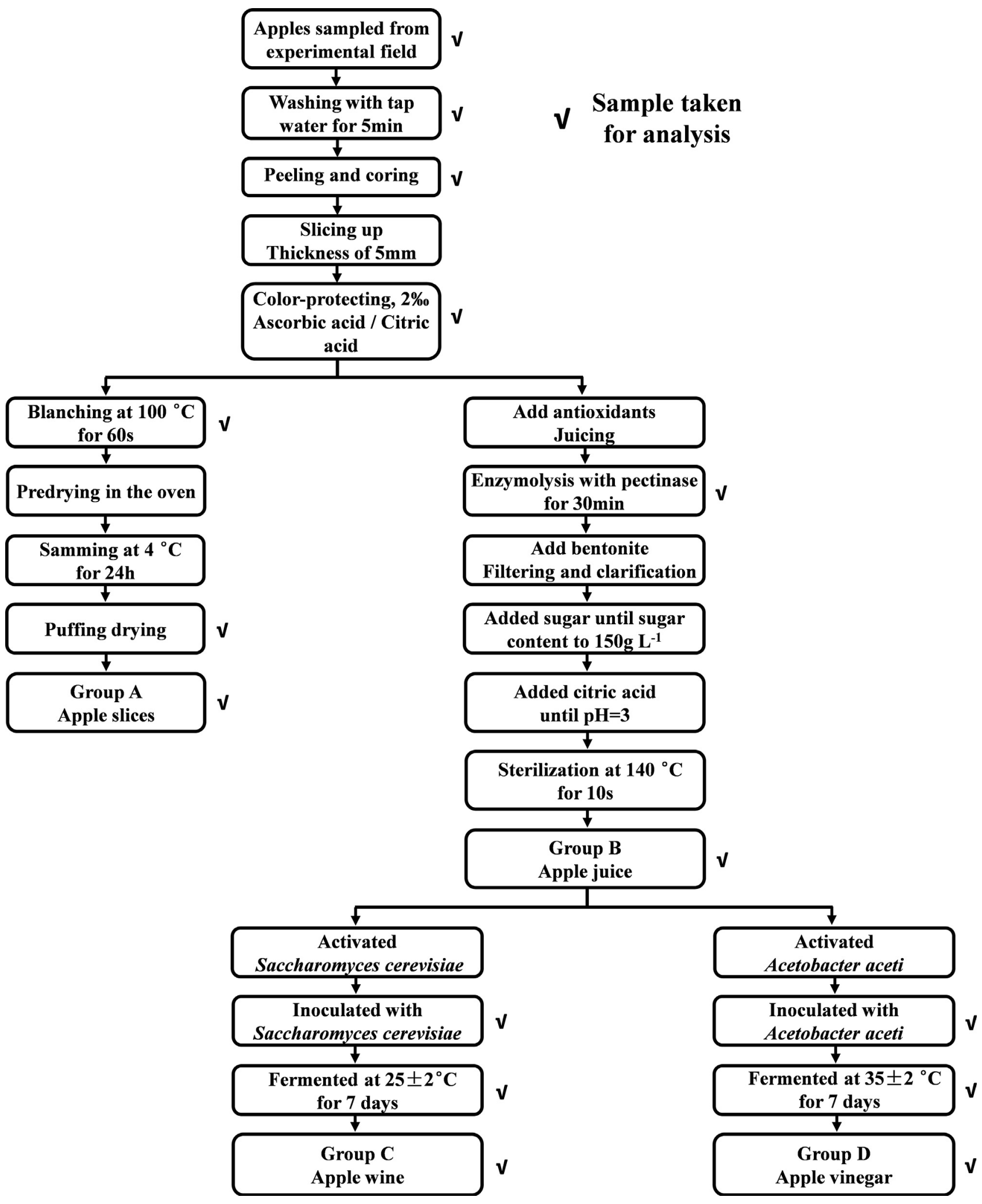

Fig. 1. Scheme for the apple products processing and sampling points used in this study.

determination $\left(\mathrm{r}^{2}\right)$ exceeding 0.9954 during solution calibration and all matrix-matched calibrations. In this experiment, the LODs of cyflumetofen enantiomers were estimated to be $2.8-4.7 \mu \mathrm{g} / \mathrm{L}$, and the corresponding LOQ was defined as the minimum standard addition concentration used for methodological validation $(10 \mu \mathrm{g} / \mathrm{L})$. The results of the recovery assay are summarized in Table 1 . Acceptable average recoveries of the enantiomers in different matrixes were within $81.1 \%$ to $119.9 \%$ at three concentration levels, except for $(+)$-cyflumetofen in apple slices with recoveries of $79.8 \%$ and $(+)$-cyflumetofen in apple juice with recoveries of $78.3 \%$. The reproducibility of the recovery studies was expressed as RSDs that ranged from $0.8 \%$ to $11.0 \%$ in all cases. The intra-day $(n=5)$ and inter-day RSDs $(n=15)$ for the 


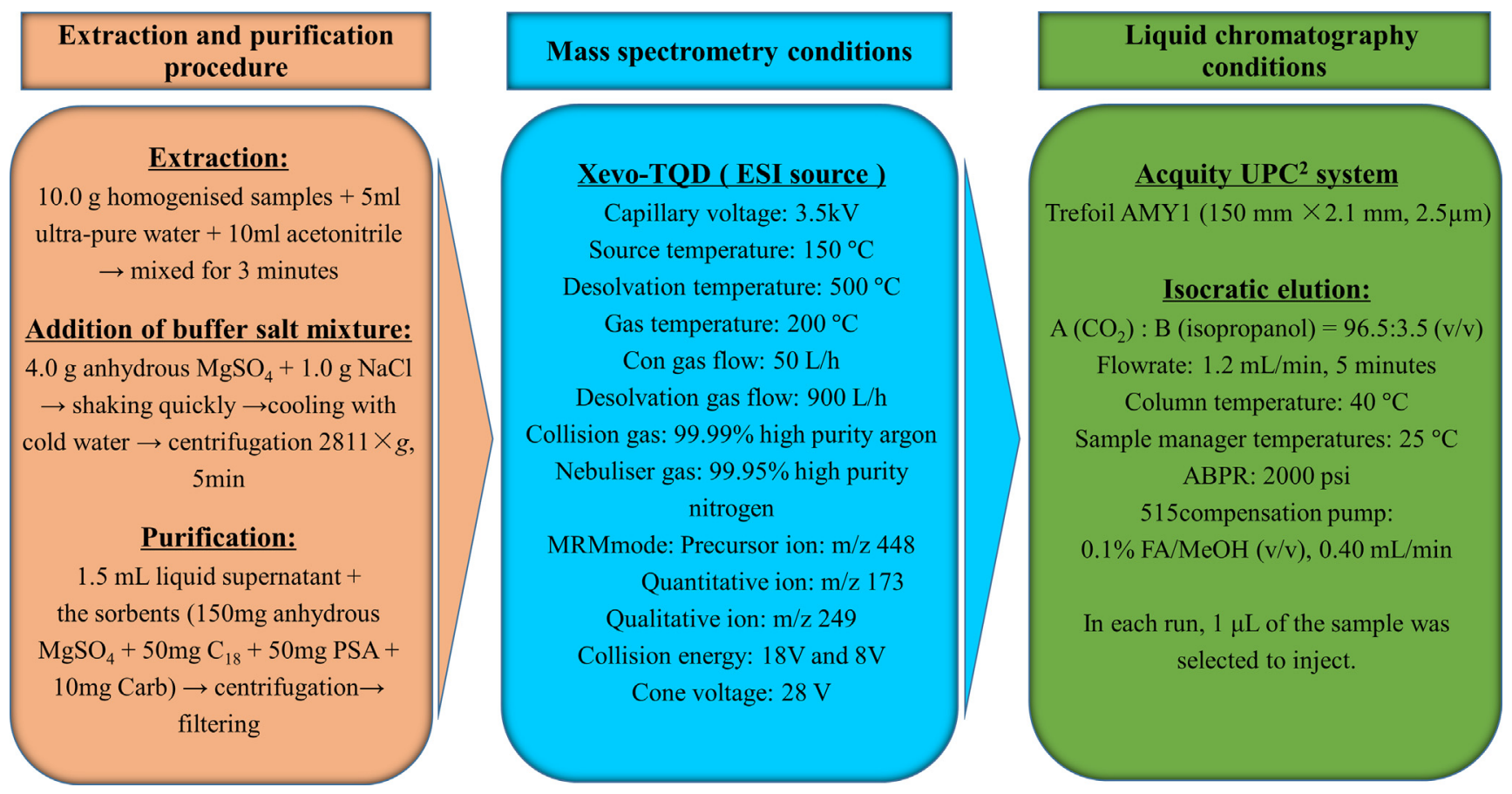

Fig. 2. Sample preparation and instrumental conditions.

Table 1

Recoveries and RSDs of cyflumetofen enantiomers in apple samples at different fortification levels $(n=5)$.

\begin{tabular}{|c|c|c|c|c|c|}
\hline \multirow[t]{2}{*}{ Matrix } & \multirow{2}{*}{$\begin{array}{l}\text { Fortification } \\
(\mu \mathrm{g} / \mathrm{kg})\end{array}$} & \multicolumn{2}{|c|}{$(+)$-cyflumetofen } & \multicolumn{2}{|c|}{$(-)$-cyflumetofen } \\
\hline & & Recoveries (\%) & RSD (\%) & Recoveries (\%) & RSD (\%) \\
\hline \multirow[t]{3}{*}{ Apple slices } & 25 & 79.8 & 9.4 & 119.8 & 3.7 \\
\hline & 250 & 109.6 & 7.4 & 119.9 & 5.5 \\
\hline & 500 & 117.2 & 5.9 & 117.0 & 1.1 \\
\hline \multirow[t]{3}{*}{ Apple juice } & 50 & 78.3 & 11.0 & 81.1 & 7.8 \\
\hline & 500 & 89.6 & 4.8 & 89.0 & 3.6 \\
\hline & 1000 & 86.7 & 2.4 & 84.9 & 3.7 \\
\hline \multirow[t]{3}{*}{ Apple wine } & 500 & 99.8 & 1.7 & 97.5 & 3.3 \\
\hline & 1000 & 98.4 & 1.6 & 97.7 & 1.6 \\
\hline & 3000 & 85.2 & 0.9 & 85.0 & 0.8 \\
\hline \multirow[t]{3}{*}{ Apple vinegar } & 500 & 104.9 & 2.2 & 104.4 & 3.4 \\
\hline & 1000 & 101.7 & 1.1 & 101.9 & 1.0 \\
\hline & 3000 & 101.4 & 3.9 & 101.8 & 3.5 \\
\hline
\end{tabular}

method ranged from $1.3 \%$ to $4.6 \%$, and $2.5 \%$ to $4.9 \%$, respectively. These results indicate that this method achieved satisfactory sensitivity, precision, and accurate quantitative data for cyflumetofen analysis in apple products, which met the guidelines of the agricultural standards of China (NY/T 788-2018).

\subsection{Effects of processing steps on the degradation of cyflumetofen residues}

The effects of specific technological procedures on cyflumetofen residue were studied. The reduction in the concentration of cyflumetofen enantiomers during apple processing is summarized in Table 2. The results showed that the degradation of $(+)$ - and $(-)$-cyflumetofen had a similar variation trend in the processing of apple slice-making and apple juice-fermenting, and both showed a decreasing trend, while the contribution rates of different operations on the degradation of pesticide were different.

\subsubsection{Washing effectiveness}

Apples were washed under flowing tap water for $5 \mathrm{~min}$ by gentle hand washing. The washed apples were dried on absorbent paper to remove excess moisture on the apple surfaces. Washing is the simplest processing method and is an initial step in the early domestic and commercial processing of apples. Multiple studies have expounded and proved the effectiveness of washing to reduce the residual pesticide content in fruits and vegetables to some extent (Aguilera, Valverde, Camacho, Boulaid, \& García-Fuentes, 2014; Kin \& Huat, 2010). In this experiment, the fresh apple sample washing resulted in $34.0 \%$ and $33.7 \%(+)$ - and (-)-cyflumetofen residue decrease, respectively. Compared with other pesticides, the effect of washing on the removal of cyflumetofen was slightly worse, mainly because of the lower watersolubility and the relatively higher octanol/water partition coefficient $\left(\log \mathrm{K}_{\mathrm{ow}}=4.3(\mathrm{OECD}, 2004)\right)$ of cyflumetofen. A higher log $\mathrm{K}_{\mathrm{ow}}$ means the pesticide is easier to penetrate the apple and more removable by the conventional washing method (Huan, Xu, Jiang, Chen, \& Luo, 2015).

\subsubsection{Peeling effectiveness}

The content of total cyflumetofen deposited on the apple peel accounted for nearly $50 \%$, and the reason for this is that the pericarp was in direct contact with the sprayed cyflumetofen and was mostly absorbed. Peeling is suggested to have a very significant impact on the 
Table 2

Reduction and PFs of cyflumetofen enantiomers of apple products following different processing procedures $(\mathrm{n}=6$ ).

\begin{tabular}{|c|c|c|c|c|c|c|}
\hline \multirow[t]{2}{*}{ Treatments } & \multicolumn{2}{|c|}{$(+)$-cyflumetofen } & \multicolumn{2}{|c|}{$(-)$-cyflumetofen } & \multirow[t]{2}{*}{ EF Values } & \multirow[t]{2}{*}{ P Values } \\
\hline & Reduction (\%) & PFs & Reduction (\%) & PFs & & \\
\hline Washing & 33.0 & 0.67 & 33.7 & 0.66 & $0.506^{* *}$ & 0.001 \\
\hline Peeling & 65.4 & 0.35 & 66.2 & 0.34 & 0.512 & 0.135 \\
\hline Color-protecting $^{1}$ & 6.7 & 0.93 & 10.6 & 0.89 & $0.522^{*}$ & 0.025 \\
\hline Color-protecting $^{2}$ & 31.8 & 0.68 & 32.7 & 0.67 & 0.500 & 0.398 \\
\hline Color-protecting ${ }^{3}$ & 25.0 & 0.75 & 25.7 & 0.74 & 0.499 & 0.446 \\
\hline Blanching & 52.1 & 0.48 & 45.3 & 0.55 & 0.488 & 0.185 \\
\hline Puffing drying & 31.2 & 0.69 & 42.1 & 0.58 & $0.532^{* *}$ & 0.001 \\
\hline Enzymolysis & 18.4 & 0.82 & 19.2 & 0.81 & 0.499 & 0.463 \\
\hline Wine Fermentation & 77.1 & 0.23 & 75.9 & 0.24 & - & - \\
\hline Vinegar Fermentation & 79.2 & 0.21 & 78.8 & 0.21 & - & - \\
\hline
\end{tabular}

*:Significant difference $(\mathrm{P}<0.05) ; * *$ :Extreme significant difference $(\mathrm{P}<0.01)$.

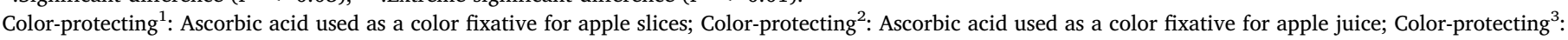
Citric acid used as a color fixative for apple juice.

degradation of cyflumetofen during the processing of apple products, with the decrease of $(+)$ - and (-)-cyflumetofen by around $65.4 \%$ and $66.2 \%$, respectively. This result is similar to other studies, where the quantity of residue removed by peeling was in the range of 60 to $100 \%$ in agricultural commodities (Cengiz, Certel, Karakaş, \& Göçmen, 2007). Jankowska et al. (2019) found that the removal of the tomato peel lowered fungicide concentrations by $68 \%$ to $87 \%$, and the pesticide residues in squeezed blackcurrants were decreased by over half when compared with the content in the raw material. Further, the results showed that peeling is more effective than washing for removal of cyflumetofen residues because the washing stage could only remove the pesticide content that is in close contact with the skin, while peeling eliminates any pesticide that has permeated the apple epidermis (Rawn et al., 2008).

\subsubsection{Color-protecting effectiveness}

In the process of making apple slices, the samples were soaked in $2 \% 0$ ascorbic acid solution for $30 \mathrm{~min}$ to protect the color and then used for subsequent processing. As shown in Table 2, residual levels of (+)and (-)-cyflumetofen were respectively reduced by $6.7 \%$ and $10.6 \%$ after color-protecting. Whereas, during the process of apple juicing, the cut apples were mixed with ascorbic acid or citric acid as a color fixative and squeezed. The color-protected apple pulp showed effective elimination of $(+)$ - and (-)-cyflumetofen with reductions of $31.8 \%$ and $32.7 \%$ when treated with ascorbic acid, respectively; meanwhile, the reductions were $25.0 \%$ and $25.7 \%$ when treated with citric acid respectively. These data indicate color protection of apple pulp is more beneficial to the degradation of pesticides than that of apple slices, which may be due to larger contact area between the color fixative and the apple itself during squeezing. In addition, ascorbic acid assisted color-protecting apparently lessened the concentrations of cyflumetofen enantiomers presented in apple pulp and was a more efficient method than using citric acid, which was probably because the acidic environment provided by ascorbic acid was not conducive to the stability of cyflumetofen with partial chemical structures being reduced in a possible reduction reaction.

\subsubsection{Blanching effectiveness}

In this study, the color-protected apple slices were blanched in $100{ }^{\circ} \mathrm{C}$ boiling water for $60 \mathrm{~s}$, which produced residue reductions of $52.1 \%$ and $45.3 \%$ respectively in the concentrations of $(+)$ - and (-)-cyflumetofen. Thermal treatments presented in the literature are characterized by different efficiencies, from the elimination of entire residues to no influence on the residue levels, while our results indicated that cyflumetofen residues were decreased during the blanching process, which was presumably because the high temperatures increased the volatilization or degradation of cyflumetofen (Han et al.,
2016). This finding is in accordance with several previous studies that demonstrated that thermal processing reduced most pesticide residues within the range of $19 \%$ to $87 \%$ in broccoli, from $43 \%$ to $93 \%$ in strawberries, and up to $98 \%$ in blackcurrants (Jankowska et al., 2019).

\subsubsection{Puffing drying effectiveness}

The temperature of the puffing dryer was adjusted to $90{ }^{\circ} \mathrm{C}$ for the first $10 \mathrm{~min}$, and therefore, the apple slices were heated evenly, and then the vacuum pump was turned on for the expansion. After the expansion was completed, the temperature was reduced to $70{ }^{\circ} \mathrm{C}$ for $2 \mathrm{~h}$, and finally, the cold circulation system and intake valve were opened for cooling and pressure reduction. The results of our study show that puffing drying exerted a little influence on the degradation of $(+)$ - and (-)-cyflumetofen, decreasing residues by $31.2 \%$ and $42.1 \%$ respectively, which may be due to the changes in the temperature and pressure. Holland et al. (1994) found that heat treatment increased the hydrolysis, degradation, and volatilization of chemicals, which leads to the progressive decrease in residue levels. In addition, the effect of pressure on the removal of pesticides has been demonstrated. Hydrostatic pressure technology could result in $75 \%$ of hydrophobic pesticide removal from cherry tomatoes and potentially replace other cleaning technologies, which may be caused by the contact/interaction between pesticides and the weakened pericarp at high pressure (Iizuka \& Shimizu, 2014). Thus, the synergistic effects of temperature and pressure are predicted to decrease pesticide residues in fruits and vegetables.

\subsubsection{Enzymolysis effectiveness}

In the apple juicing process, pectinase was added to hydrolyze the pulp for $30 \mathrm{~min}$. The enzymolysis was showed to lead to a reduction in the levels of $(+)$ - and (-)-cyflumetofen in the hydrolyzed pulp by $18.4 \%$ and $19.2 \%$, respectively. As previously mentioned, Han et al. (2013) found that enzyme treatment brought about the independent reduction in the residues of spirotetramat and its metabolite by approximately 24\%. Moreover, Nagayama (1997) confirmed that the pectin in fruits could adsorb pesticides partly, which resulted in a lower concentration of pesticide residue.

\subsubsection{Fermentation effectiveness}

As a technique for processing and preserving food, fermentation has been widely used at domestic and commercial levels, and has played a significant role in reducing pesticide residues in different kinds of processing. In this study, sterilized apple juice was fermented at $25{ }^{\circ} \mathrm{C}$ for the production of apple wine, and $35^{\circ} \mathrm{C}$ for apple vinegar for 7 days in two closed fermentation tanks. The amounts of $(+)$ - and (-)-cyflumetofen were reduced by $75.9 \%, 77.1 \%$ in apple wine, and by $78.8 \%$ and $79.2 \%$ in apple vinegar, respectively, showing similar variation 

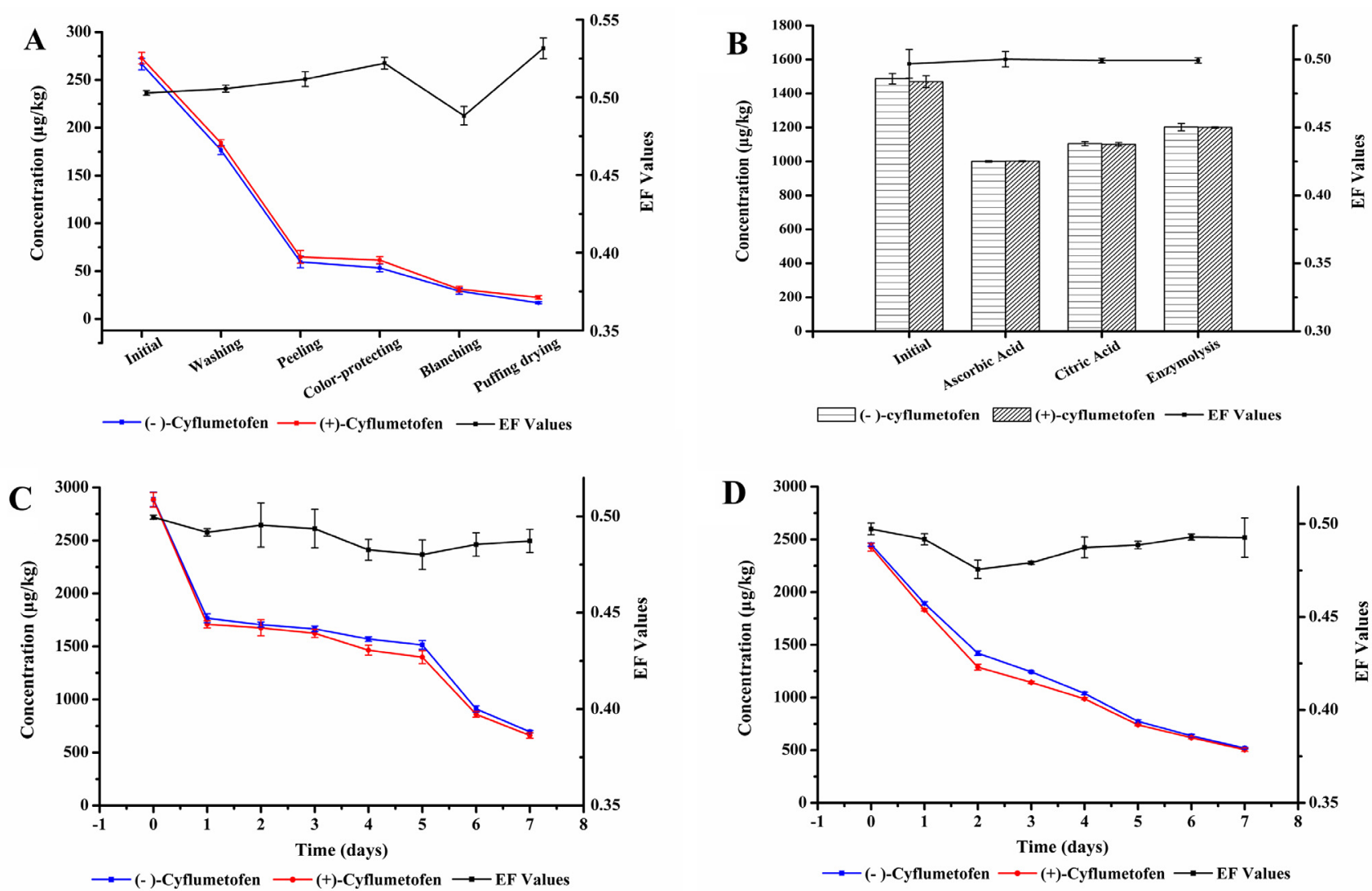

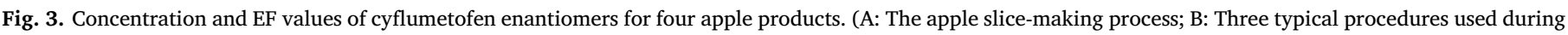
the apple juicing process; C: Apple wine fermentation; D: Apple vinegar fermentation).

tendency (Table 2 and Fig. 3C, D). The highest degradation rate was obtained for cyflumetofen in apple products following fermentation. The concentration of (+)-cyflumetofen was the same as that of $(-)$-cyflumetofen at the beginning of fermentation, which demonstrated that no selective degradation of cyflumetofen in the previous stages of apple juicing occurred. However, with elapsed time, the concentration of enantiomers gradually decreased, and (+)-cyflumetofen was preferentially degraded during fermentation resulting in relative enrichment of (-)-cyflumetofen in apple wine and vinegar. The results obtained confirmed that fermentation of apple products reduced pesticide levels, which is possibly attributable to the microbial degradation induced by the activity of the microorganism. In this study, $S$. cerevisiae and $A$. aceti were added to provide the necessary biological system for brewing apple wine and apple vinegar, respectively. Cabras and Angioni (2000) found that pesticides could affect the activity of yeasts, and conversely, yeasts reduced pesticide residues or absorbed pesticides partly in the fermentation process. Similar findings were obtained by Navarro, Perez, Navarro, Mena, and Vela (2006), who showed that there was no residue of dinitroaniline herbicides in fresh beer fermented with bottom yeasts. Therefore, focusing on the variations of the pesticide residues during apple processing is essential for ensuring future food safety.

\subsection{Enantioselective behavior of cyflumetofen in the course of apple processing}

$\mathrm{EF}$ values were calculated to demonstrate the stereoselective dissipation of cyflumetofen enantiomers in the processing of apple products. The EF values of different processes (Table 2 and Fig. 3) inconspicuously changed, varying gradually from 0.512 in peeling, to 0.500 and 0.499 in color-protecting by addition of ascorbic acid and citric acid in the apple pulp, respectively, to 0.488 in blanching and 0.499 in enzymolysis. The degradation rates of the two enantiomers of cyflumetofen were found to be stably maintained in the above processes, which may be because there was no chiral environment that could bring about stereoselective dissipation of cyflumetofen. Color-protection of apple slices using ascorbic acid, with an $\mathrm{EF}=0.522$, degraded enantiomers selectively to a certain extent ( $\mathrm{p}<0.05$ ). The EF value of puffing drying increased from the initial value of 0.500 to 0.532 , illustrating enantioselectivity with (-)-cyflumetofen being degraded faster than $(+)$-cyflumetofen $(\mathrm{p}<0.01)$. This finding may be because of the different effects of temperature and pressure on the physical and chemical properties of the two enantiomers. In general, there are differences between enantiomers in the space structure, biological activity, and degradation behavior. When high temperatures or high pressures are encountered, the enantiomer with an unstable spatial configuration is more likely to be destroyed and degraded.

Generally, enantiomers of chiral pesticides tend to exhibit different degradation rates when exposed to biosystems. As shown in Fig. 3(C, D), the original EFs of the cyflumetofen enantiomers were close to 0.5, which showed that there was no enantioselectivity in the apple juice sample. During fermentation, the degradation rate of the enantiomers was different; the EF values fluctuated slightly; however, the equilibrium was approximately 0.5 . The corresponding degradation kinetics and half-lives of cyflumetofen enantiomers are presented in Table 3. The reduction of cyflumetofen enantiomers in fermentation was in accordance with first-order kinetics, in which the half-lives of $(-)$ - and (+)-cyflumetofen were 4.25 and 4.05 days in apple wine-making, and 3.18 and 3.19 days in apple vinegar-making respectively. The half-life of cyflumetofen in wine-fermentation was approximately 1.3 times that observed in vinegar-fermentation, indicating that the biological environment provided by $A$. aceti was more conducive to the elimination of cyflumetofen. In addition, in the fermentation of apple wine or vinegar, the enantiomers exhibited enantioselective degradation to 
Table 3

Degradation kinetic constant $(\mathrm{k})$, half-life $\left(\mathrm{T}_{1 / 2}\right)$ and correlation coefficient $\left(\mathrm{R}^{2}\right)$ values for the dissipation of cyflumetofen enantiomers during fermentation.

\begin{tabular}{lllll}
\hline Sample & Compounds & $\mathrm{K}^{*} 10^{-2}\left(\right.$ day $\left.^{-1}\right)$ & $\mathrm{T}_{1 / 2}$ (day) & $\mathrm{R}^{2}$ \\
\hline Apple wine & ( -)-cyflumetofen & 17.1 & $4.25^{\mathrm{a}}$ & 0.8689 \\
& (+)-cyflumetofen & 16.3 & $4.05^{\mathrm{b}}$ & 0.8443 \\
\multirow{2}{*}{ Apple vinegar } & ( -)-cyflumetofen & 21.8 & $3.18^{\mathrm{C}}$ & 0.9949 \\
& (+)-cyflumetofen & 21.7 & $3.19^{\mathrm{C}}$ & 0.9874 \\
\hline
\end{tabular}

a-c: Values with different letters are significantly different $(P<0.05)$.

different degrees, with (+)-cyflumetofen degrading preferentially (Supplementary Table 1). Selective degradation behavior varied with fermentation time, mainly in the metaphase of the two processes. Previous studies have illustrated the stereoselective behavior of chiral pesticides during fermentation (Lu, Shao, Dai, Diao, \& Chen, 2016; Pan et al., 2018). It is preliminarily judged that the existence of microorganisms such as yeasts and associated enzymes play a crucial role in chiral recognition and stereoselectivity of chiral compounds. In addition, this phenomenon may be related to the intrinsic properties and toxicity of enantiomers in microorganisms. Existing studies have shown that the enantiomers of cyflumetofen usually showed different toxicities (Sun et al., 2016) and the $\mathrm{LC}_{50}$ (Lethal Concentration 50) of the l-isomer and d-isomer of cyflumetofen was $339 \mathrm{mg} / \mathrm{L}$ and $706 \mathrm{mg} / \mathrm{L}$, respectively, in an acute toxicity test of animals, which indicates the toxicity of the 1-isomer was significantly higher than that of the d-isomer. Further study is required to illustrate the mechanism of selective degradation of chiral pesticides during fermentation. Hence, the data obtained will supply a reliable reference for the risk assessment of cyflumetofen during fermentation.

\subsection{Processing factors}

The calculated PFs of the cyflumetofen enantiomers during apple processing were presented in Table 2. The values were less than 1 , which indicated that the individual processing stage could reduce remarkably cyflumetofen in the processed apple commodity. Washing was found to be effective for the removal of pesticide residues in raw fruits and vegetables, and the research is extensively reported (Chen, Liu, \& Jiao, 2016; Huan et al., 2015; Kin \& Huat, 2010). In our study, the PF value of washing on apples reached 0.66, despite the low water solubility of cyflumetofen. Beyond that, the low PF values were obtained following peeling, with PFs of $(+)$ - and (-)-cyflumetofen after peeling being 0.35 and 0.34 respectively, which manifested that peeling was an effective approach for pesticide residue removal. This result was similar to previous studies, which demonstrated that peeling could reduce the residues of racemate and enantiomers of zoxamide to some extent, with PFs ranging from 0.057 to 0.060 (Pan et al., 2018). Such findings are not unique, Liu, Dong, Chen, et al. (2016) calculated the PF for cyflumetofen after peeling as 0.28 . Hence, for consumers, peeling should be performed first to minimize pesticide concentrations in food. The lowest PF value of all data acquired, approximately 0.21 by fermentation, indicated this process played the most crucial role in decreasing residues of cyflumetofen effectively from apples. The PF for apple vinegar was lower than that in apple wine, presumably because of the diverse $\mathrm{pH}$ environment during the fermentation of apple wine or vinegar (Singh \& Kulshrestha, 2000). Zhao, Liu, Wu, Xue, and Hou (2017) found that the PFs of triadimefon reached 0.12 and 0.09 in the process of jujube wine and vinegar fermentation, respectively, which suggested that the fermentation process had a remarkable effect on the degradation of triadimefon. The results of our study illustrated that the consumption of apples after simple home preparation might be safer for consumers. Nevertheless, a limitation should be highlighted regarding the use of the above PFs for apple products, in that the formation of cyflumetofen metabolites might produce greater toxicity. There is still a lack of data regarding concentration of these metabolites. Consequently, it is particularly necessary to pay close attention to the impact of processes not only regarding pesticide residues but also the formation of their metabolites to further improve food safety in future research.

\section{Conclusions}

In this study, the stereoselective behavior of cyflumetofen enantiomers during apple products processing was carefully investigated. Typical processing treatments could affect the degradation rate of cyflumetofen to different degrees, where the PFs of all processing steps are generally less than 1 . In addition, a similar dissipation trend of cyflumetofen enantiomers was observed. Particularly, the enantioselectivity of the two enantiomers was discussed in relation to the fermentation process. Enantioselective behavior was identified in two processing groups, with $(+)$-cyflumetofen preferentially degraded to a certain extent, which contributed to the relative enrichment of (-)-cyflumetofen in apple wine and apple vinegar. Consequently, it is indispensable to consider these findings when ensuring the security of processed food. To conclude, these results are may be helpful to ascertain the safety of cyflumetofen in apple products and to allow the refinement of dietary risk assessments after food processing. Nevertheless, in the future, more attention should be paid to optimizing processing technology in a manner that brings about considerable pesticide residue degradation but preserves most of the elementary nutrient content during food processing.

\section{CRediT authorship contribution statement}

Rui Quan: Conceptualization, Software, Validation, Formal analysis, Data curation, Writing - original draft, Writing - review \& editing. Minmin Li: Conceptualization, Methodology, Validation, Formal analysis, Writing - review \& editing, Funding acquisition. Yongguo Liu: Formal analysis, Writing - review \& editing. Nuo Jin: Investigation, Validation, Methodology, Writing - review \& editing. Jia Zhang: Validation, Data curation, Methodology. Ruixing Li: Investigation, Data curation, Software. Fengzhong Wang: Resources, Supervision, Project administration, Funding acquisition. Zhidong Wang: Writing review \& editing. Frédéric Francis: Writing - review \& editing. Zhiqiang Kong: Methodology, Software, Formal analysis, Data curation, Writing - review \& editing, Funding acquisition. Bei Fan: Conceptualization, Methodology, Resources, Supervision, Project administration, Funding acquisition, Writing - review \& editing.

\section{Declaration of Competing Interest}

The authors declare that they have no known competing financial interests or personal relationships that could have appeared to influence the work reported in this paper.

\section{Acknowledgments}

This study was supported by the National Natural Science Foundation of China (31872006 and 31671942), the Beijing Nova Program of Science and Technology (Z191100001119121) and the National Key Research and Development Program of China (2017YFC1600600). One of the authors, Minmin Li, would like to acknowledge the financial support from the China Scholarship Council and also thank the University of Liège-Gembloux Agro-Bio Tech (MOE11-BE1A20131371N), and more specifically the research platform Agriculture Is Life for the funding for this research project. We would like to thank Editage (www.editage.cn) for English language editing. 


\section{Ethical approval}

This article does not contain any studies with human participants or animals performed by any of the authors.

\section{Appendix A. Supplementary data}

Supplementary data to this article can be found online at https:// doi.org/10.1016/j.foodchem.2020.126687.

\section{References}

Aguilera, A., Valverde, A., Camacho, F., Boulaid, M., \& García-Fuentes, L. (2014). Household processing factors of acrinathrin, fipronil, kresoxim-methyl and pyridaben residues in green beans. Food Control, 35, 146-152.

Anastassiades, M., Lehotay, S., \& Štajnbaher, D. (2002). Quick, easy, cheap, effective, rugged, and safe (QuEChERS) approach for the determination of pesticide residues. Pesticide Protocols, 19, 239-261.

Cabras, P., \& Angioni, A. (2000). Pesticide residues in grapes, wine, and their processing products. Journal of Agricultural and Food Chemistry, 48, 967-973.

Cengiz, M. F., Certel, M., Karakaş, B., \& Göçmen, H. (2007). Residue contents of captan and procymidone applied on tomatoes grown in greenhouses and their reduction by duration of a pre-harvest interval and post-harvest culinary applications. Food Chemistry, 100, 1611-1619.

Chen, X., Dong, F., Liu, X., Xu, J., Li, J., Li, Y., ... Zheng, Y. (2013). Chiral determination of a novel acaricide cyflumetofen enantiomers in cucumber, tomato, and apple by HPLC. Journal of Separation Science, 36, 225-231.

Chen, W., Liu, Y., \& Jiao, B. (2016). Dissipation behavior of five organophosphorus pesticides in kumquat sample during honeyed kumquat candied fruit processing. Food Control, 66, 87-92.

Geng, Y., Zhao, L., Zhao, J., Guo, B., Ma, P., Li, Y., \& Zhang, T. (2014). Development of a supercritical fluid chromatography-tandem mass spectrometry method for the determination of lacidipine in beagle dog plasma and its application to a bioavailability study. Journal of Chromatography B, 945, 121-126.

Guo, J., Li, M., Liu, Y., Wang, F., Kong, Z., Sun, Y., ... Liu, J. (2018). Residue and dietary risk assessment of chiral cyflumetofen in apple. Molecules, 23, 1060.

Han, Y., Huang, B., Liu, S., Zou, N., Yang, J., Zhong, Z., ... Pan, C. (2016). Residue levels of five grain-storage-use insecticides during the production process of sorghum distilled spirits. Food Chemistry, 206, 12-17.

Han, Y., Xu, J., Dong, F., Li, W., Liu, X., Li, Y., ... Zheng, Y. (2013). The fate of spirotetramat and its metabolite spirotetramat-enol in apple samples during apple cider processing. Food Control, 34, 283-290.

Holland, P., Hamilton, D., Ohlin, B., \& Skidmore, M. (1994). Effects of storage and pro cessing on pesticide residues in plant products. Pure and Applied Chemistry, 66, 335-356.

Huan, Z., Xu, Z., Jiang, W., Chen, Z., \& Luo, J. (2015). Effect of Chinese traditional cooking on eight pesticides residue during cowpea processing. Food Chemistry, 170, $118-122$.

Iizuka, T., \& Shimizu, A. (2014). Removal of pesticide residue from cherry tomatoes by hydrostatic pressure (Part 2). Innovative Food Science \& Emerging Technologies, 26 34-39.

Jankowska, M., Łozowicka, B., \& Kaczyński, P. (2019). Comprehensive toxicological study over 160 processing factors of pesticides in selected fruit and vegetables after water, mechanical and thermal processing treatments and their application to human health risk assessment. Science of the Total Environment, 652, 1156-1167.

Keikotlhaile, B. M., Spanoghe, P., \& Steurbaut, W. (2010). Effects of food processing on pesticide residues in fruits and vegetables: A meta-analysis approach. Food and Chemical Toxicology, 48, 1-6.

Kin, C. M., \& Huat, T. G. (2010). Headspace solid-phase microextraction for the evaluation of pesticide residue contents in cucumber and strawberry after washing treatment. Food Chemistry, 123, 760-764.

Kong, Z., Li, M., Chen, J., Gui, Y., Bao, Y., Fan, B., ... Dai, X. (2016). Behavior of fieldapplied triadimefon, malathion, dichlorvos, and their main metabolites during barley storage and beer processing. Food Chemistry, 211, 679-686.

Li, M., Liu, X., Dong, F., Xu, J., Qin, D., \& Zheng, Y. (2012). Determination of cyflumetofen residue in water, soil, and fruits by modified quick, easy, cheap, effective, rugged, and safe method coupled to gas chromatography/tandem mass spectrometry. Journal of Separation Science, 35, 2743-2749.

Li, M., Liu, Y., Fan, B., Lu, J., He, Y., Kong, Z., ... Wang, F. (2015). A chemometric processing-factor-based approach to the determination of the fates of five pesticides during apple processing. LWT-Food Science and Technology, 63, 1102-1109.

Liu, N., Dong, F., Chen, Z, Xu, J., Liu, X, Duan, L. Zheng, Y. (2016). Distribution behaviour of acaricide cyflumetofen in tomato during home canning. Food Additives and Contaminants - Part A Chemistry, Analysis, Control, Exposure and Risk Assessment, 33, 824-830.

Liu, N., Dong, F., Xu, J., Liu, X., Chen, Z., Pan, X., ... Zheng, Y. (2016). Enantioselective separation and pharmacokinetic dissipation of cyflumetofen in field soil by ultraperformance convergence chromatography with tandem mass spectrometry. Journal of Separation Science, 39, 1363-1370.

Liu, Y., Zhang, X., Liu, C., Yang, R., Xu, Z., Zhou, L., ... Lei, H. (2015). Enantioselective and synergetic toxicity of axial chiral herbicide propisochlor to SP2/0 myeloma cells. Journal of Agricultural and Food Chemistry, 63, 7914-7920.

Liu, M.q., \& Zhao, J.y. (2018). Export status, competitiveness and promotion countermeasures of apple products in China. Food and Nutrition in China, 24, 47-51.

Lu, Y., Shao, Y., Dai, S., Diao, J., \& Chen, X. (2016). Stereoselective behavior of the fungicide Benalaxyl during grape growth and the wine-making process. Chirality, 28, 394-398.

Lunder, S. (2016). EWG news and analysis. https://www.ewg.org/foodnews/summary. php\#dirty-dozen.

Nagayama, T. (1997). Decrease in organic solvent extractable ethion by grapefruit pectin during processing. Journal of Agricultural and Food Chemistry, 45, 4856-4860.

Navarro, S., Perez, G., Navarro, G., Mena, L., \& Vela, N. (2006). Decay of dinitroaniline herbicides and organophosphorus insecticides during brewing of lager beer. Journal of Food Protection, 69, 1699-1706.

OECD, T. (2004). 117: Partition Coefficient (n-octanol/water). HPLC Method.

OECD. (2008). Test No. 508: Magnitude of the Pesticide Residues in Processed Commodities, OECD Guidelines for the Testing of Chemicals, Section 5

Pan, X., Dong, F., Liu, N., Cheng, Y., Xu, J., Liu, X., ... Zheng, Y. (2018). The fate and enantioselective behavior of zoxamide during wine-making process. Food Chemistry, $248,14-20$.

Porter, P. S., Ward, R. C., \& Bell, H. F. (1988). The detection limit. Environmental Science \& Technology, 22, 856-861.

Rasmusssen, R., Poulsen, M. E., \& Hansen, H. (2003). Distribution of multiple pesticide residues in apple segments after home processing. Food Additives and Contaminants, 20, 1044-1063.

Rawn, D. F., Quade, S. C., Sun, W.-F., Fouguet, A., Bélanger, A., \& Smith, M. (2008). Captan residue reduction in apples as a result of rinsing and peeling. Food Chemistry, 109, 790-796.

Scholz, R., Donkersgoed, G., Herrmann, M., Kittelmann, A., Schledorn, M., Graven, C., ... Michalski, B. (2018). Database of processing techniques and processing factors compatible with the EFSA food classification and description system FoodEx 2 Objective 3: European database of processing factors for pesticides in food. EFSA Supporting Publication, 15, 1-50.

Seydim, A. C., Guzel-Seydim, Z. B., Doguc, D. K., Savas, M. C., \& Budak, H. N. (2016). Effects of grape wine and apple cider vinegar on oxidative and antioxidative status in high cholesterol-fed rats. Functional Foods in Health and Disease, 6, 569-577.

Singh, S. B., \& Kulshrestha, G. (2000). Effect of ph on the hydrolysis of chlorimuron-ethyl, a sulfonyl urea herbicide. Pesticide Research Journal, 12, 235-238.

Sun, D., Pang, J., Zhou, Z., \& Jiao, B. (2016). Enantioselective environmental behavior and cytotoxicity of chiral acaricide cyflumetofen. Chemosphere, 161, 167-173.

Wang, P., Li, M., Liu, X., Xu, J., Dong, F., Wu, X., \& Zheng, Y. (2016). Degradation of cyflumetofen and formation of its main metabolites in soils and water/sediment systems. Environmental Science and Pollution Research, 23, 23114-23122.

Yoshida, T., Ikemi, N., Takeuchi, Y., Ebino, K., Kojima, S., Chiba, Y., ... Harada, T. (2012). A repeated dose 90-day oral toxicity study of cyflumetofen, a novel acaricide, in rats. The Journal of Toxicological Sciences, 37, 91-104.

Zhao, L., Liu, F., Wu, L., Xue, X., \& Hou, F. (2017). Fate of triadimefon and its metabolite triadimenol in jujube samples during jujube wine and vinegar processing. Food Control, 73, 468-473. 